\title{
弱磁场强化零价铁对水中污染物的去除效能及其作用机制
}

\author{
李锦祥 秦荷杰张雪莹 关小红* \\ (同济大学环境科学与工程学院 污染控制与资源化国家重点实验室 上海 200092)
}

\begin{abstract}
摘要 作为一种环境友好、价格低廉的水处理药剂, 零价铁(ZVI)的低反应活性已成为基于 ZVI 水处理技术工程应用的 一个重要障碍. 如何提高 ZVI 对污染物的去除已成为目前研究的焦点, 作者课题组近年来围绕探究弱磁场(WMF)对 ZVI 除有毒污染物的正面效应及其作用机制展开了系列研究. 该系列工作明确了 WMF 能够提高 ZVI 对有毒金属离子 的去除速率, 探明了 WMF 强化 ZVI 除污染的作用机制主要是通过磁场梯度力实现的. 为进一步探索磁场对零价铁除 污染的正面效应，作者提出通过磁场预磁化来提高 ZVI 对水中污染物的反应活性，且考察了该方法强化不同来源零价 铁去除不同污染物的广谱性. 利用 WMF 强化 ZVI 对水中污染物的去除具有高效、操作简单、低成本、适用广泛以及 无二次污染等优点, 因而利用 WMF 是一种应用前景广阔的提高 ZVI 活性的水处理技术.

关键词 零价铁; 弱磁场; 预磁化; 金属离子; 磁场梯度力; 剩磁
\end{abstract}

\section{Improving the Reactivity of Zerovalent Iron toward Various Contaminants by Weak Magnetic Field: Performances and Mechanisms}

\author{
Li, Jinxiang Qin, Hejie Zhang, Xueying Guan, Xiaohong* \\ (State Key Laboratory of Pollution Control and Resources Reuse, College of Environmental Science and Engineering, \\ Tongji University, Shanghai 200092)
}

\begin{abstract}
Zero-valent iron (ZVI), a simple but amazingly versatile material, has low intrinsic reactivity toward various contaminants as documented from laboratory studies as well as field demonstrations, which poses potential limitations to its practical application in environmental remediation. Although many methods have been developed to improve the reactivity of ZVI in the literature, high costs, significant work-load, and complex operations may inhibit the application of these methods. We pioneered the research in employing weak magnetic field (WMF) to accelerate the removal of various metal(loid)s, including $\mathrm{Se}(\mathrm{IV}) / \mathrm{Se}(\mathrm{VI}), \mathrm{As}(\mathrm{V}) / \mathrm{As}(\mathrm{III}), \mathrm{Sb}(\mathrm{V}), \mathrm{Cu}(\mathrm{II}) / \mathrm{EDTA}-\mathrm{Cu}(\mathrm{II})$, and $\mathrm{Cr}(\mathrm{VI})$ by pristine ZVI (Pri-ZVI) and/or aged ZVI. The rate constants of metal(loid)s sequestration by Pri-ZVI or aged ZVI were increased by $1.1 \sim 383.7$ folds due to the application of WMF. Furthermore, WMF could be employed to improve the removal of organic contaminants by ZVI activated $\mathrm{H}_{2} \mathrm{O}_{2}$ or persulfate because of the accelerated ZVI corrosion in the presence of WMF. The superimposed WMF had negligible influence on the apparent activation energy of metal(loid)s removal by ZVI, indicating that WMF accelerated metal(loid)s removal by ZVI but did not change the mechanisms. The XAFS, XRD, and XPS analysis confirmed that the application of WMF did not change the mechanisms of metal(loid)s removal but accelerated the transformation (reduction or oxidation) of contaminants. Electrochemical analysis showed that the accelerated ZVI corrosion in the presence of WMF was ascribed to the enhanced mass transfer. We further identified the relative contribution of Lorentz force $\left(F_{\mathrm{L}}\right)$ and magnetic gradient force $\left(F_{\triangle \mathrm{B}}\right)$ in the enhancing effect of WMF. It suggested that $F_{\triangle \mathrm{B}}$ rather than $F_{\mathrm{L}}$ was the major driving force for the observed WMF effect on the enhanced reactivity of ZVI. Moreover, we proposed to apply premagnetization to increase the reactivity of ZVI toward As(III) sequestration taking advantage of the magnetic memory of ZVI, i.e., the remanence of ZVI. In addition, the premagnetized ZVI (Mag-ZVI) samples from different origins were applied to enhance the removal of various oxidative contaminants [such as azo dyes, $\mathrm{As}(\mathrm{III}), \mathrm{Pb}(\mathrm{II}), \mathrm{Cu}(\mathrm{II}), \mathrm{Se}(\mathrm{IV}), \mathrm{Ag}(\mathrm{I})$ and $\mathrm{Cr}(\mathrm{VI})$ ] under well-controlled experimental conditions. The rate constants of contaminants removal by premagnetized ZVI samples were $1.2 \sim 12.2$ folds greater than those by Pri-ZVI samples. As a chemical- and energy-free method, improving the reactivity of ZVI by either WMF superimposition or premagnetization treatment is novel and promising.
\end{abstract}

Keywords zerovalent iron; weak magnetic field; premagnetization; metal(loid)s; magnetic field gradient force; remanence

\section{1 引言}

尽管早在 20 世纪 90 年代前后研究人员就已发表了 关于利用零价铁(ZVI)去除水中的氯代有机物[如三氯乙
烯(TCE)、1,1,2,2-四氯乙烯(PCE)]和无机物[如 Cr(VI)] 的研究论文 ${ }^{[1,2]}$, 并且随之在 1991 年首次成功地将零价 铁渗透反应墙(PRB)应用于受 TCE 和 PCE 污染的地下水

\footnotetext{
*E-mail: guanxh@tongji.edu.cn; Tel.: +86-21-65980956; Fax: +86-21-65986313

Received January 7, 2017; published February 20, 2017.

Project supported by the National Natural Science Foundation of China (Nos. 51478329, 21522704, and U1532120).

项目受国家自然科学基金(Nos. 51478329, 21522704, U1532120)资助.
} 
原位修复中, 但是直到 1994 年美国和加拿大的两组研 究人员分别发表了利用零价铁去除水中的卤代有机物 的论文后 ${ }^{[3,4]}$, 零价铁才以其操作简单、运行成本低廉、 环境绿色友好等优势迅速在水污染治理中受到重视. 到 目前为止, 零价铁已广泛应用于 PRB 地下水修复、工业 废水处理以及受重金属污染的地下水为水源的饮用水 处理中, 美国环保署(USEPA)更是在 2002 年将基于零价 铁的 PRB 技术列为一种标准的地下水污染修复技术 ${ }^{[5]}$, 零价铁水处理技术已在水污染治理领域中扮演着越来 越重要的角色.

尽管零价铁技术已在实际中得到了较为广泛的应 用, 该技术仍存在着许多缺点 ${ }^{[6]}$, 其归纳如下: (1)零价 铁颗粒表面存在的固有钝化膜使得其初始反应活性较 低; (2)反应活性随腐蚀产物的生成会进一步降低; (3)零 价铁对某些惰性污染物去除能力非常弱; (4)零价铁对污 染物的靶向去除能力非常弱; 5某些污染物在高浓度条 件下会导致零价铁的钝化等. 正是由于该技术局限性的 存在, 因此当前零价铁多用作 PRB 的填充材料来原位 修复水体, 此时零价铁与污染物之间能有足够的时间接 触、反应. 然而在将零价铁用于工业废水处理或受污染 地下水的异位处理时, 反应时间过长将导致单位时间内 产水量较小, 反应器体积过大. 因此, 寻找一种简单有 效的提高零价铁活性的方法来拓宽零价铁的应用领域 已成为当前的研究热点.

针对零价铁技术所存在的缺陷, 学者们开展了大量 的研究工作试图找到有效的措施来提高零价铁的反应 性能, 这些尝试的发展历程如图 1 所示. 目前, 具体措 施主要集中在以下几个方面: 酸洗预处理法 ${ }^{[4]}$ 、合成纳 米级零价铁法 ${ }^{[7]}$ 、超声预处理法 ${ }^{[8]}$ 、合成铁系双金属材

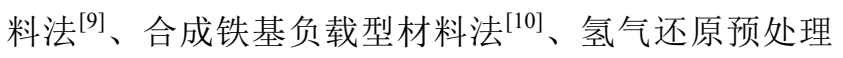
法 $^{[11] 、 \text { 紫外光照法 }}{ }^{[12]}$ 、微波法 ${ }^{[13]}$ 、外加金属离子法 等 $[14,15]$.

尽管这些方法能够一定程度上改善零价铁对污染 物的去除活性, 但是它们仍然存在着在实际应用方面的 局限性. 例如, 酸洗预处理法对于零价铁活性改善效果 一般; 而氢气还原预处理法操作比较复杂, 而且成本较 高; 合成的纳米零价铁虽然能够显著改善零价铁活性, 但是其操作复杂、成本很高, 而且还存在潜在的生态毒 性、颗粒团聚以及无法大批量工程应用等缺点; 超声、 电解、电还原、紫外光照法等方法需要额外的能耗, 难 以大规模实际应用; 而合成铁系双金属材料则面临着贵 金属泄露对环境产生二次污染的风险. 目前提高零价铁 活性的方法大部分是基于通过破除零价铁表面的钝化 膜或提高其表面积来实现, 然而考虑到零价铁的腐蚀必 将产生大量的铁氧化物, 短时间内活性的提高最终也必 将导致更厚的氧化膜生成, 因此依靠改善零价铁颗粒表 面性质来提高零价铁活性的思路也许并不合理.

铁水体系去除有毒金属离子的反应属于典型的非

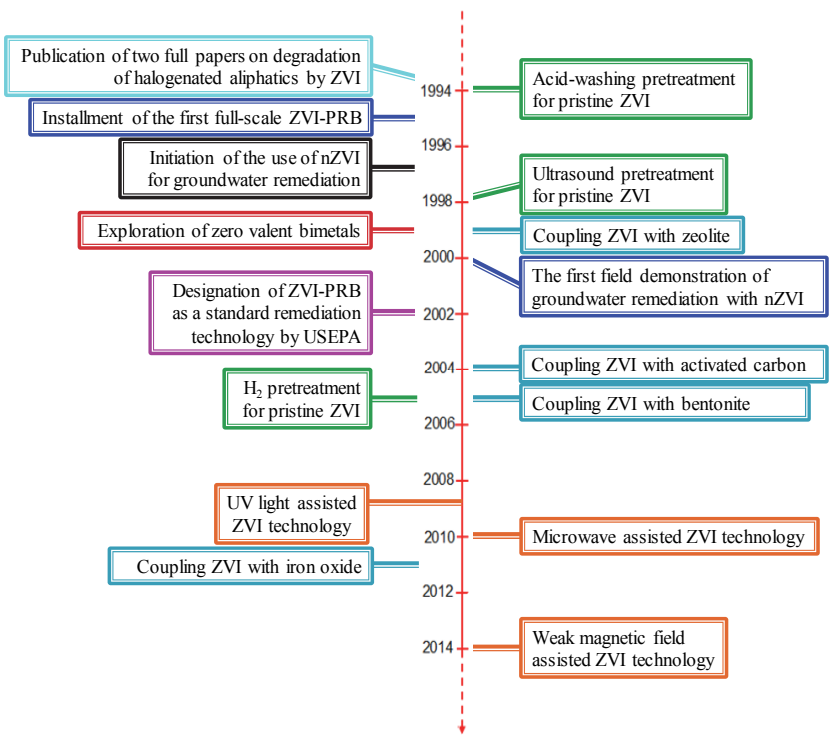

图 1 零价铁水处理技术发展简史 $(1994 \sim 2014 \text { 年 })^{[6]}$

Figure 1 The major events in the development of ZVI technology in the past two decades ${ }^{[6]}$

均相界面化学反应, 如图 2 所示, 其固液界面处的反应 过程主要包括以下几个步骤: (1)溶液中有毒金属离子通 过扩散层向零价铁的表面扩散; (2)扩散到零价铁表面的 有毒金属离子被零价铁吸附; (3)被吸附的有毒金属离子 在零价铁上发生反应并生成被零价铁表面所吸附的产 物; (4)产物脱附由扩散层进入溶液. 尽管反应步骤(3)是 有毒金属离子迁移、转化的核心步骤(经此步骤其被转 化为无毒或低毒性的溶解态或固态物质), 但是反应步 骤(1)、(2)和(4)对有毒金属离子的去除速率有着一定程 度的影响，是提高零价铁技术去除有毒金属离子反应活 性的重要考虑因素 ${ }^{[4]}$.

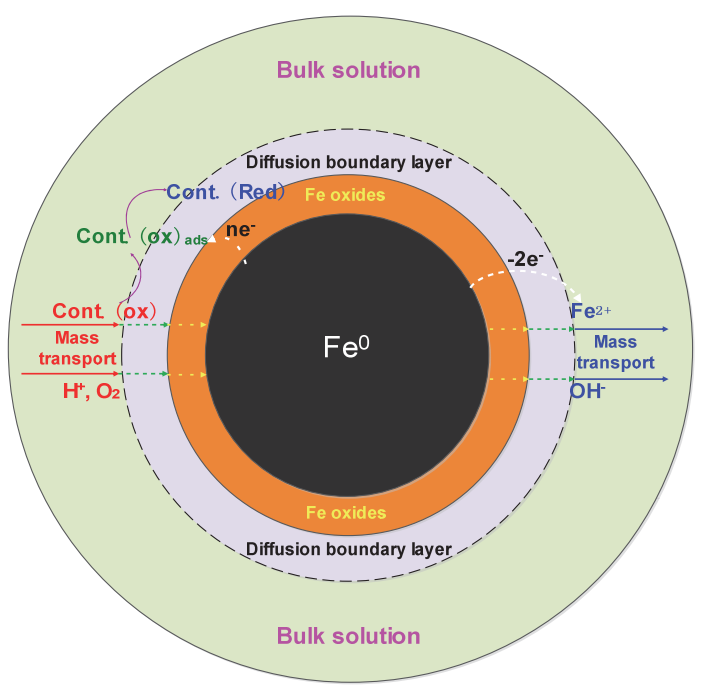

图 $2 \mathrm{Fe}^{0} / \mathrm{H}_{2} \mathrm{O}$ 体系除污染界面反应过程示意图 ${ }^{[6]}$

Figure 2 Illustration of the process of contaminants sequestration by the $\mathrm{Fe}^{0} / \mathrm{H}_{2} \mathrm{O}$ system ${ }^{[6]}$ 
归纳来说, 污染物、 $\mathrm{DO} 、 \mathrm{H}^{+}$要与零价铁反应需要 其扩散通过扩散层、氧化膜进入零价铁内部, 而反应之 后则需要对应的反应产物(如亚铁离子、氢气等)沿着反 应路径相反的方向扩散回溶液, 扩散过程的快慢很大程 度上影响着零价铁与污染物的反应速率. 考虑到零价铁 腐蚀必然会导致铁氧化膜的生成, 那么也许可以通过加 速溶液中反应物及产物的传质或者改变腐蚀产物在零 价铁颗粒表面的分布来实现零价铁去除污染物活性的 提高. 基于此, 显然搅拌可以加速溶液中相关物质之间 的传质, 其研究结果也表明搅拌速度的提高能够改善零 价铁去除污染物的反应速率 ${ }^{[16]}$. 然而, 搅拌只是加速了 体系中反应物由溶液向铁颗粒表面边界层扩散的这一 传质过程, 搅拌并不能为反应物或产物穿越此扩散层提 供驱动力 ${ }^{[17]}$, 也无法对反应物或产物在铁颗粒表面氧 化膜内的传质提供正面效应.

\section{2 弱磁场强化零价铁高效除污染技术}

自 20 世纪初 Faraday 发现磁场能够影响化学反应至 今, 国内外关于磁场对零价铁除污染物的影响的研究少 之又少. 其中, Kim 等 ${ }^{[18]}$ 研究发现放置于反应器下部的 磁铁所提供的磁场能够提高零价铁对 4-氯酚的反应效 率, 其认为零价铁去污染速率的提高主要是由于磁场能 够促进氧气与零价铁之间的传质, 从而加速 $\mathrm{Fe}^{0}-\mathrm{O}_{2}$ 体系 产生具有强氧化能力 $\cdot \mathrm{OH}$ 的速率. 此外, Jiang 等 ${ }^{[19]}$ 和 Ambashta 等 ${ }^{[20]}$ 也都发现利用直流电磁场能够改善零价 铁对污染物的反应活性. 但是, 这些相关论文没有深入 研究磁场形式、磁场强度等对零价铁去除污染物的影响, 未把磁场对零价铁腐蚀过程的影响与污染物的去除联 系起来, 并未明确磁场影响零价铁除污染物的原因, 而 且这几个课题组的相关研究没有形成体系.

2013 年初, 我们在一次烧杯试验中发现, 将铁水体 系除污染的摚拌方式从机械搅拌转换为磁力摚拌后, 零 价铁的腐蚀速率明显加快, 其对水中 $\mathrm{Se}(\mathrm{IV})$ 的去除速率 得到了显著地提升. 针对这一奇异的实验现象, 我们进 行了深入的文献查阅和进一步的实验探索, 证实零价铁 活性的显著提高是由于磁力搅拌器内置磁铁以及转子 内部磁铁共同提供的弱磁场引起的. 由此, 我们提出利 用弱磁场(WMF)驱动零价铁对水中有毒金属离子的去 除这一研究方向, 揭开了弱磁场零价铁水处理技术的研 究序幕. 随之, 研究人员通过利用两个硬币大小的磁铁 提供弱磁场(反应器内的磁场强度为 $1 \sim 10 \mathrm{mT}$ ), 开展了 一系列的研究工作, 其概括如下.

\section{1 弱磁场对原始零价铁除污染的提升作用}

针对弱磁场对零价铁除有毒金属离子反应活性的 影响, 其研究结论归纳如下: 弱磁场能够强化不同来源 的原始零价铁对水中不同金属离子的去除性能. Liang 等 ${ }^{[21,22]}$ 发现弱磁场能够显著提高原始 7.4-mic ${ }^{\text {\#零价铁对 }}$ 水中 $\operatorname{Se}(\mathrm{IV})$ 和 $\mathrm{Se}(\mathrm{VI})$ 的去除性能. 对于 $\mathrm{Se}(\mathrm{IV})$ 的去除,
弱磁场能够有效拓宽零价铁除 $\operatorname{Se}(\mathrm{IV})$ 的 $\mathrm{pH}$ 工作范围, 且随着 $\mathrm{Se}(\mathrm{IV})$ 的初始浓度提高, WMF 对 ZVI 除 Se(IV) 的强化作用逐渐减弱; Sun 等 ${ }^{[23]}$ 同样发现弱磁场对原始 Jinshan 零价铁去除水中 $\mathrm{As}(\mathrm{III})$ 和 $\mathrm{As}(\mathrm{V})$ 的反应活性具 有促进作用, 通过表征该体系的 $\mathrm{pH}$ 变化、 $\mathrm{Fe}^{2+}$ 释放以及 借助 $\mathrm{Fe}$ 边 X 射线精细结构谱(XANES)分析腐蚀产物生 成及演变, 证实弱磁场能够促进零价铁的腐蚀, 从而改 善了体系对污染物的反应活性. 并且, 通过去除实际地 下水中砷的研究进一步证实了弱磁场零价铁水处理技 术具有实际应用前景. 此外, 弱磁场能够强化原始

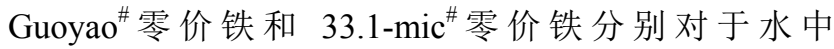
$\mathrm{Cu}(\mathrm{II}) / \mathrm{EDTA}-\mathrm{Cu}(\mathrm{II})^{[24,25]}$ 和 $\mathrm{Cr}(\mathrm{VI})^{[26]}$ 的去除.

为进一步量化弱磁场对零价铁反应活性的增效作 用, 定义零价铁在有弱磁场条件下除污染反应速率常数

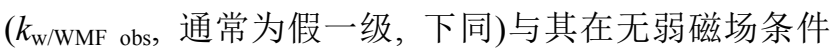
下反应速率常数 $\left(k_{\mathrm{w} / \mathrm{o}} \mathrm{WMF}\right.$ obs $)$ 的比值 $\left(R_{\mathrm{WMF}}\right)$, 即为弱磁场 对零价铁反应活性的增效因子. 如图 3 所示, 弱磁场能 够将零价铁除 $\mathrm{Cu}(\mathrm{II})$ 的速率常数提高至 $10.8 \sim 383.7$ 倍 ${ }^{[25]}$; 对于 $\mathrm{Cr}(\mathrm{VI})$, 弱磁场不仅能够有效缓解高浓度 $\mathrm{Cr}(\mathrm{VI})$ 对零价铁的致钝效应, 且弱磁场对零价铁除 $\mathrm{Cr}(\mathrm{VI})$ 反应速率常数的提高倍数为 $1.1 \sim 5.9$ 倍 ${ }^{[26]}$; 同样 地， $\mathrm{Li}$ 等 ${ }^{[27]}$ 发现利用弱磁场也能够显著改善高浓度 $\mathrm{Sb}(\mathrm{V})$ 对零价铁的钝化效应. 在 $\mathrm{Sb}(\mathrm{V})$ 初始浓度为 $5.0 \sim$ $40.0 \mathrm{mg} \cdot \mathrm{L}^{-1}$ 的条件下, 弱磁场对零价铁除 $\mathrm{Sb}(\mathrm{V})$ 反应速 率的提高倍数为 $5.6 \sim 7.7$ 倍, 且其能将零价铁对 $\mathrm{Sb}(\mathrm{V})$ 的去除容量从 $18.1 \mathrm{mg} \mathrm{Sb}(\mathrm{V}) \mathrm{g}^{-1} \mathrm{Fe}$ 提高至 $39.2 \mathrm{mg}$ $\mathrm{Sb}(\mathrm{V}) \mathrm{g}^{-1} \mathrm{Fe}$. 此外, $\mathrm{Xu}$ 等 ${ }^{[28]}$ 发现弱磁场能够促进零价 铁对 $\mathrm{Sb}(\mathrm{III})$ 的去除速率常数提高倍数为 $6.0 \sim 8.0$ 倍.

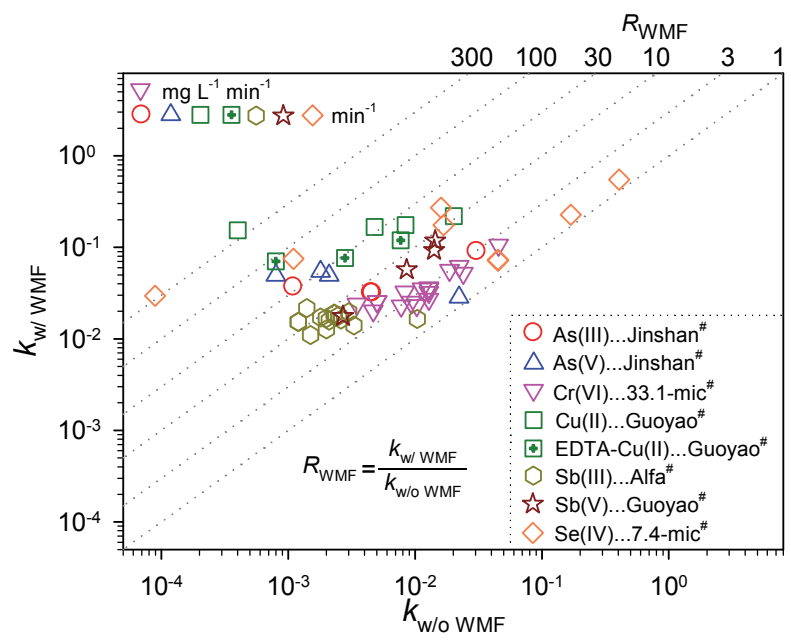

图 3 弱磁场对零价铁除有毒金属离子反应速率及促进作用概括 Figure 3 Summary of the performances of WMF for enhancing metal(loid)s sequestration by ZVI or aged ZVI from different origins

总体来看，弱磁场对不同来源零价铁除不同污染物 反应速率常数的提高倍数呈现了一个复杂的趋势, 但其 整体上位于 $1.1 \sim 88.0$ 倍这一区间范围，只有两个值远 远超出于此(即, 弱磁场对原始 7.4-mic ${ }^{\#}$ 零价铁除水中 
$\mathrm{Se}(\mathrm{IV})$ 反应速率常数提高倍数为 330.4 倍 ${ }^{[21]}$, 以及上述 提及的弱磁场对原始 Guoyao 零价铁除水中 $\mathrm{Cu}(\mathrm{II})$ 反应 速率常数提高倍数为 383.7 倍 ${ }^{[25]}$ ), 而这可能主要与零价 铁的钝化程度以及污染物是否为顺磁性有关.

\section{2 弱磁场对老化铁反应活性的提升作用}

此外, 弱磁场还能够强化不同老化条件的老化铁 (AZVI) 对水中金属离子的去除性能. 研究结果表明, 一方面弱磁场能够提高老化时间介于 $6 \sim 60 \mathrm{~h}$ 的老化铁 反应活性, $\mathrm{Se}(\mathrm{IV})$ 的去除速率相比无磁场时提高了 $10.0 \sim 100.0$ 倍 ${ }^{[29]}$; 另一方面, 对于含有不同性质针化膜 的老化铁而言, 弱磁场对其也具备活化作用 ${ }^{[30]}$. 为进一 步明确弱磁场对零价铁除污染的促进行为, 我们系统地 概括了弱磁场对原始零价铁以及已老化零价铁除污染 物活性的促进作用. 结果表明, WMF 对不同来源原始零 价铁除不同污染物反应活性促进作用 $\left(R_{\mathrm{WMF}}\right)$ 的中值为 15.9 倍, 而对应的老化铁体系 $R_{\mathrm{WMF}}$ 的中值为 24.6 倍. 归 纳而言, 相比于原始零价铁, 弱磁场对已老化零价铁对 有毒金属离子去除性能的促进作用更为显著, 这一定程 度上说明弱磁场对零价铁除污染物的强化作用和已老 化零价铁颗粒表面铁氧化物对污染物的富集作用对于 $\mathrm{Fe}^{0} / \mathrm{H}_{2} \mathrm{O}$ 体系除污染物存在协同效应.

\section{3 弱磁场对零价铁活化过硫酸盐/双氧水去除有机污 染物的加速作用}

弱磁场不仅能够强化基于零价铁对有毒金属离子 的还原、吸附/共沉淀去除, 还能够强化基于零价铁对有 机物降解去除的高级氧化技术. Xiong 等 ${ }^{[31]}$ 以对硝基酚 (4-NP) 作为目标污染物, 系统地考察了在不同的铁投 量、 $\mathrm{H}_{2} \mathrm{O}_{2}$ 投加量、 $\mathrm{pH}_{\mathrm{ini}}$ 以及磁场强度条件下, 弱磁场对 $\mathrm{Fe}^{0} / \mathrm{H}_{2} \mathrm{O}_{2}$ 体系降解 4-NP动力学的影响. 研究发现, 弱磁 场不仅能够拓宽零价铁的有效 $\mathrm{pH}$ 工作范围(从 $3.0 \sim 5.0$ 拓宽到 $3.0 \sim 6.0$ ), 还能够加速零价铁对 4-NP 的降解速 率, 降解速率提高倍数为 $1.5 \sim 8.2$ 倍, 如图 4 所示. 此 外, 该研究还发现弱磁场没有影响体系的自由基种类, 只提高了自由基 $[\cdot \mathrm{OH}$ 和 $\mathrm{Fe}(\mathrm{IV})]$ 的累积产生量, 且证实 弱磁场能够促进体系对 4-NP 的矿化. 对于 $\mathrm{Fe}^{0} / \mathrm{H}_{2} \mathrm{O}_{2}$ 体 系, 章北平研究组 ${ }^{[32]}$ 的研究结果同样表明, 弱磁场不仅 能够加速体系除污染的传质过程, 而且还能够影响铁颗 粒表面形貌、缩短反应过程的初始平台期.

此外, 研究还发现弱磁场能够显著提高零价铁活化 过硫酸盐(PS)技术对有机污染物的氧化降解. 对于咖啡 因、苯并三唑和敌草隆, 弱磁场的增效因子(即反应速率 的提高倍数, $R_{\mathrm{WMF}}$ ) 分别为 $8.3 、 3.1$ 和 13.1 倍 ${ }^{[33]}$. 相比于 $\mathrm{Fe}^{0} / \mathrm{H}_{2} \mathrm{O}_{2}$ 体系, 弱磁场对 $\mathrm{Fe}^{0} / \mathrm{PS}$ 体系降解有机物的促进 作用更为显著. 如图 4 所示, 弱磁场对零价铁活化 PS 降 解染料橙黄 $\mathrm{G}(\mathrm{OG})$ 反应速率的提高倍数为 $6.4 \sim 29.0$ 倍, 而这主要是由于 PS 体系反应过程中 $\mathrm{pH}$ 下降以及弱磁 场的增效作用两者之间存在协同效应. 利用弱磁场强化

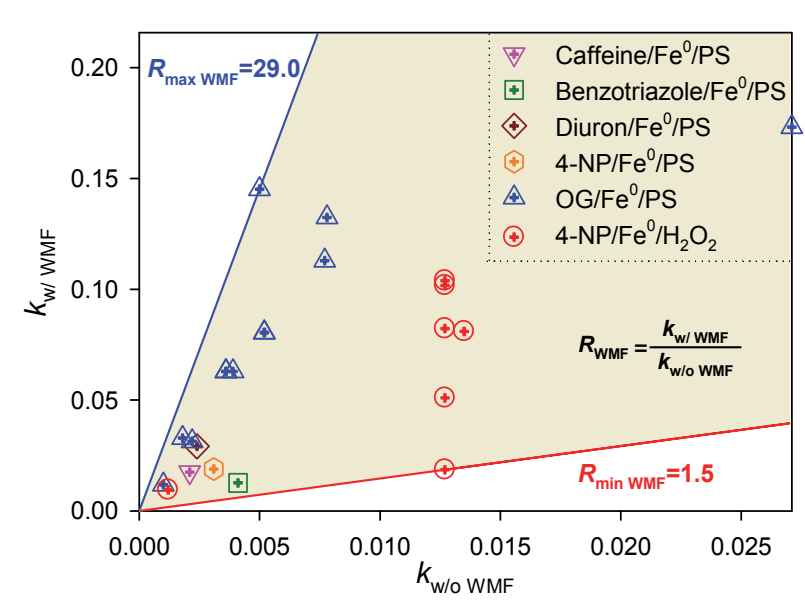

图 4 弱磁场对零价铁活化过硫酸盐/双氧水体系除有机污染物反应 速率及促进作用概括

Figure 4 Summary of the performances of WMF for enhancing various organic compounds degradation by the $\mathrm{Fe}^{0} / \mathrm{H}_{2} \mathrm{O}_{2}$ and/or $\mathrm{Fe}^{0} / \mathrm{PS}$ system

基于零价铁对污染物的去除是一种新颖有效、环境友 好、应用前景广阔的水污染控制技术 ${ }^{[28,32]}$, 已受到国内 外研究者们的广泛关注.

\section{4 弱磁场对零价铁除有毒金属离子反应机理的影响}

针对弱磁场对零价铁除有毒金属离子反应机理的 影响, Liang 等 ${ }^{[29]}$ 通过 X 射线近边结构谱(XANES 谱)分 析表明，在不同反应条件下, WMF 的存在能够加速零价 铁除 $\mathrm{Se}(\mathrm{IV})$ 的还原、吸附抑或是吸附一还原过程; 零价铁 去除 $A s(I I I)$ 的机理包含先氧化后吸附以及先吸附再在 固相中氧化两种去除机制, 研究表明弱磁场的施加能加 快溶液中 $\mathrm{As}(\mathrm{III})$ 的氧化速度, 且在初始 $\mathrm{pH}$ 为 $5.0 \sim 9.0$ 时其还能加速固相中 $\mathrm{As}(\mathrm{III})$ 的氧化 ${ }^{[23]}$. 此外, $\mathrm{Li}$ 等 ${ }^{[27]}$ 通 过傅里叶变换后径向结构图谱(FT)和 X射线精细结构谱 (EXAFS)表征考察了弱磁场对于 $\mathrm{Sb}-\mathrm{Fe}$ 配位距离和配位 数的影响, 并且进一步证实了 WMF 仅仅是加速了 $\mathrm{Sb}(\mathrm{V})$ 的去除而并未影响 $\mathrm{Sb}(\mathrm{V})$ 的去除机制. Feng 等 ${ }^{[26]}$ 也证实 $\mathrm{WMF}$ 并没有改变零价铁与 $\mathrm{Cr}(\mathrm{VI})$ 的反应活化能, 而 Jiang 等 ${ }^{[25]}$ 通过电化学实验从电化学腐蚀的角度明确 了弱磁场并没有影响铁电极腐蚀的电子转移过程, 只是 加速了其电化学腐蚀的传质过程. 综上所述, WMF 能够 加速零价铁除污染物的反应过程, 并未改变零价铁对污 染物的去除机理.

\section{5 弱磁场对零价铁反应活性的强化作用机制}

要明确弱磁场对零价铁除污染活性的强化作用机 制, 首先要清楚磁场与零价铁之间的关系. 众所周知, 零价铁是一种铁磁性的材料, 其在外置磁场条件下能够 产生一个沿着外加磁场方向的、远高于外加磁场强度的 感应磁场. 且由于零价铁颗粒表面曲折蜿蜒的特点, 使 得其表面磁感应强度分布存在明显的磁场梯度. 而这二 者(即, 磁场强度和磁场梯度)能够分别通过形成洛伦兹 力效应 $\left(F_{\mathrm{GMF}}\right)$ 和磁场梯度力效应 $\left(F_{\Delta \mathrm{B}}\right)$ 影响铁水体系中离 
子的迁移, 最终影响体系的反应活性 ${ }^{[34]}$.

具体地说, 洛伦兹力指的是当一个带电荷的粒子作 切割磁感线运动的时候, 会受到一个垂直于运动方向的 力, 此即为洛伦兹力 ${ }^{[35]}$. 洛伦兹力能够影响铁水体系中 带电粒子的运动方向, 使其在零价铁颗粒表面形成一个 扫洗的效应, 进而使得颗粒表面的扩散层厚度减小, 从 而对体系中的相关物质的传质提供了一个正面效应, 最 终改善零价铁与污染物的反应活性(如图 5) ${ }^{[25]}$.

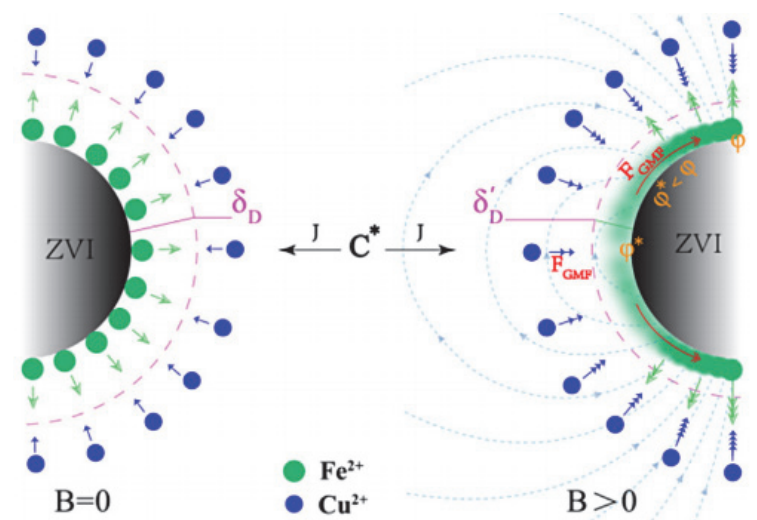

图 5 弱磁场对零价铁除铜离子体系中铁颗粒表面扩散层厚度的影响 示意图 $^{[25]}$

Figure 5 Schematic illustration of the role of WMF in the thickness of diffusion boundary layer in $\mathrm{Fe}^{0} / \mathrm{Cu}^{2+}$ system ${ }^{[25]}$

而磁场梯度力能够作用于顺磁性的离子 ${ }^{[36,37]}$, 使其 由磁感应强度低的位置向磁感应较高的位置方向迁移, 导致零价铁的腐蚀产物在零价铁颗粒表面呈现非均匀 分布, 并同时形成了点蚀效应, 从而来改善、维持零价 铁在除污染过程中的反应活性.

通过考察弱磁场对铁丝腐蚀产生的铁氧化物在其 表面分布的影响, 可以得出, 相比于无弱磁场存在时铁 腐蚀产物在铁丝表面的均匀分布, 弱磁场的存在能够使 得腐蚀产物更倾向分布于铁丝磁感应强度高的位置, 这 一定程度上说明了磁场梯度力在其中的参与作用 ${ }^{[29]}$. 由于磁场梯度力的作用, 铁丝腐蚀产生的顺磁性 $\mathrm{Fe}^{2+}$ 以 及 $\mathrm{Fe}^{3+}$ 被作用向磁感应强度更高的位置迁移, 因而使得 铁腐蚀产物在铁丝表面非均匀分布. 此外, Jiang 等 ${ }^{[25]}$ 以 顺磁性 $\mathrm{Cu}^{2+}$ 为探针离子, 考察了弱磁场对 $\mathrm{Cu}^{2+}$ 与铁丝 反应后 $\mathrm{Cu}$ 元素在铁丝上分布的影响. 同样地, 该研究 发现弱磁场的存在能够使得顺磁性 $\mathrm{Cu}^{2+}$ 更趋向于向磁 感应强度高的铁丝位置迁移, 继而再发生氧化还原反 应, 而这也进一步地说明弱磁场的强化作用机制一定程 度上与磁场梯度力有关.

在初步探讨了弱磁场强化作用机制后, 仍然还需要 我们进一步明确上述提及的洛伦兹力和磁场梯度力到 底在弱磁场强化作用中扮演了一个什么样的角色. 到底 是磁场梯度力起主要作用还是洛伦兹力起主要作用? 换句话说，这二者作用力效应在弱磁场强化作用的机制 中扮演的角色需要进一步量化明确.
$\mathrm{Li}$ 等 ${ }^{[38]}$ 研究发现, 尽管磁场强度的提升意味着洛 伦兹力的提高, 但是其并不会影响非铁磁性零价锌的反 应活性(即意味着洛伦兹力对零价铁反应体系影响较 弱), 且弱磁场对零价铁除污染物的促进作用随着铁颗 粒粒径的减小而逐渐显著. 此外, 通过将弱磁场对零价 铁活性的强化作用分别与洛伦兹力和磁场梯度力的固 有系数作相关性分析发现, 弱磁场强化作用与前者无明 显关系, 而与磁场梯度力的系数呈现非常明显的线性相 关. 为进一步阐明弱磁场对零价铁腐蚀活性的作用机 制, 研究团队利用上海光源 BL08U1A 和 BL14W1 的两 组线站对零价铁的腐蚀行为进行了系统地表征, 发现弱 磁场能够使得零价铁的腐蚀产物在零价铁颗粒表面呈 现非常明显的非均匀的分布, 而这就是源于磁场梯度力 作用于顺磁性离子 (如零价铁腐蚀产生的 $\mathrm{Fe}^{2+} 、 \mathrm{Fe}^{3+}$ ), 使 其由磁感应强度较低的位置向磁感应强度较高的位置 方向迁移. 因而可以证实, 弱磁场对零价铁反应活性的 强化作用机制主要与磁场梯度力有关, 如图 6 所示. 此 外, 最近 $\mathrm{Xu}$ 等 ${ }^{[28]}$ 的研究结果表明对反应体系投加顺磁 性 $\mathrm{Fe}^{2+}$ 后, 弱磁场对零价铁除污染反应活性的提升作用 更为显著, 这进一步说明了弱磁场对零价铁除污染物的 强化作用主要是通过磁场梯度力作用于体系的顺磁性 离子而实现的.

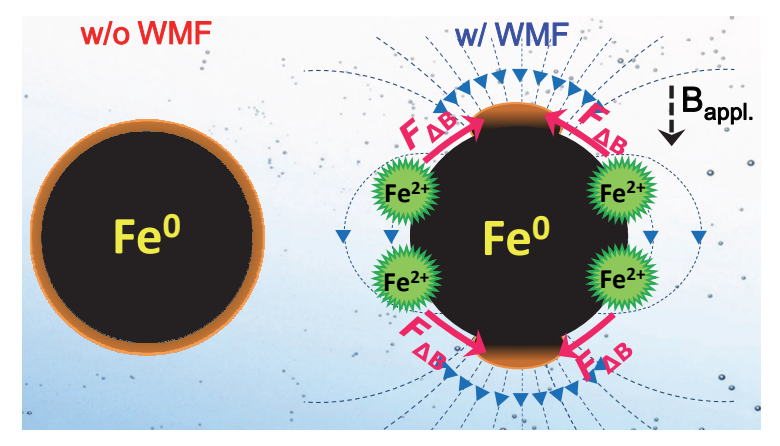

图 6 弱磁场对零价铁活性作用机制示意图 ${ }^{[38]}$

Figure 6 Schematic illustration of the mechanism for the improved reactivity of $\mathrm{ZVI}$ by $\mathrm{WMF}^{[38]}$

\section{3 磁场预磁化强化零价铁高效除污染技术}

如前文所言弱磁场能够驱动零价铁对水中有毒金 属离子的高效去除，但考虑到该技术的实际运行仍可能 存在一定的缺陷. 首先, 磁场反应器的构建会导致工程 成本的增加; 其次, 磁场强度过高会导致零价铁颗粒的 团聚进而导致零价铁除污染活性降低, 而磁场强度过低 又不能完全发挥弱磁场对零价铁活性的正面效应, 因此 如何合理地外置弱磁场将成为弱磁场零价铁水处理技 术实际应用的 “瓶颈”.

考虑到弱磁场零价铁技术实际应用存在的局限性, 且为进一步探索磁场对零价铁除污染的正面效应, 考虑 利用零价铁的铁磁记忆特性 ${ }^{[39,40]}$, 而这一 “记忆” 的磁 场有可能会改善零价铁活性. 因此, 通过磁场预磁化的 
手段就有可能改善零价铁去除水中有毒金属离子的性 能.

\section{1 磁场预磁化对零价铁除污染的提升作用}

考虑到零价铁可能在不同磁场强度环境下其 “记 忆” 的磁场强度不同, 而磁场强度又能影响零价铁除污 染活性, 因此我们首先考察了不同预磁化强度对零价铁 除水中 $\mathrm{As}(\mathrm{III})$ 反应动力学的影响 ${ }^{[41]}$. 如图 7 所示, 随着 预磁化强度的提高(即预磁化的磁场强度从 0 提高至 500 $\mathrm{mT}$ ), 零价铁对水中总 As 的反应速率常数从 0.0159 $\mathrm{min}^{-1}$ 逐渐升高至 $0.0667 \mathrm{~min}^{-1}$. 磁场预磁化不仅有助于 加速氧化水溶态的 As(III), 还能够在固液表面将吸附态 的 $\mathrm{As}(\mathrm{III})$ 加速氧化为 $\mathrm{As}(\mathrm{V})$, 进而证实、解释了零价铁 以吸附-氧化和氧化一吸附的反应机理实现对水中 $\mathrm{As}(\mathrm{III})$ 的去除 ${ }^{[23]}$.

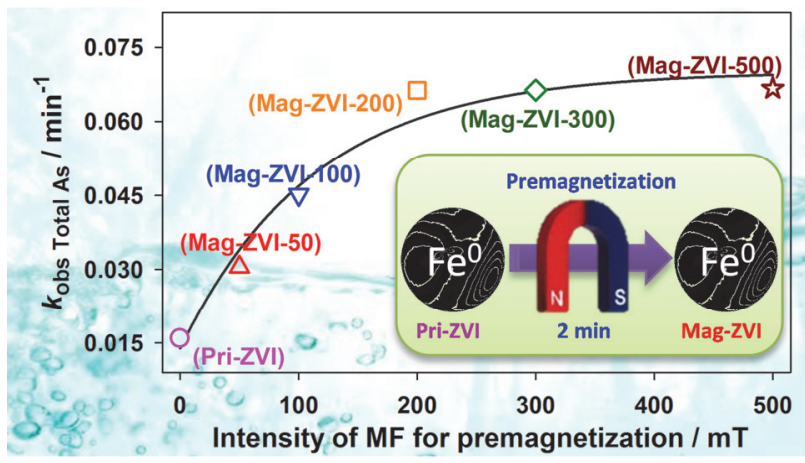

图 7 磁场预磁化强化零价铁去除水中 $\mathrm{As}(\mathrm{III})$ 示意图 ${ }^{[4]}$

Figure 7 Schematic illustration of the improved reactivity of ZVI toward As(III) by taking advantage of its magnetic memory ${ }^{[41]}$

预磁化能够显著提高零价铁对水中污染物的去除, 其作用机制是由于预磁化后的零价铁存在剩磁，因而其 颗粒表面能够产生感应磁场, 感应磁场能通过磁场梯度 力作用顺磁性离子 (如腐蚀过程中产生的 $\mathrm{Fe}^{2+} 、 \mathrm{Fe}^{3+}$ ), 使 得零价铁的腐蚀加速, 从而实现零价铁对水中污染物的 高效去除. 为进一步探究零价铁预磁化技术实际应用的

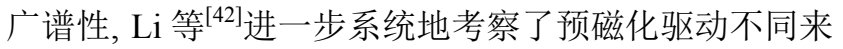
源的零价铁对水中不同污染物强化去除性能及影响机 制, 并以此利用对应反应体系拟合得出线性方程预测相 关铁粉去除某一污染物的性能. 如图 8 所示, 预磁化能 够强化 8 种不同来源的铁材料对水中染料 AR27(苋菜 红)、 $\mathrm{Pb}^{2+} 、 \mathrm{Cu}^{2+} 、 \mathrm{SeO}_{3}^{2-} 、 \mathrm{Ag}^{+}$和 $\mathrm{HCrO}_{4}^{-}$的去除, 且其 对零价铁去除污染物的反应速率 $\left(k_{\mathrm{obs}}\right)$ 提高倍数为 $1.2 \sim$ 12.2. 因而, 利用磁场预磁化对于零价铁除污染具有广 谱性. 周明华课题组 ${ }^{[43]}$ 在我们研究的基础上, 考察了预 磁化对零价铁活化过硫酸盐去除水中有机污染物 2,4-二 氯苯酚(2,4-DCP)、对氯苯酚(4-CP)和氯苯 $(\mathrm{CB})$, 结果表 明 Mag-ZVI/PS 体系对污染物的降解去除速率是 Pri-ZVI/PS 体系的 1.9 5.2 倍, 这证实了预磁化促进零 价铁腐蚀进而促进零价铁除污染的有效性.

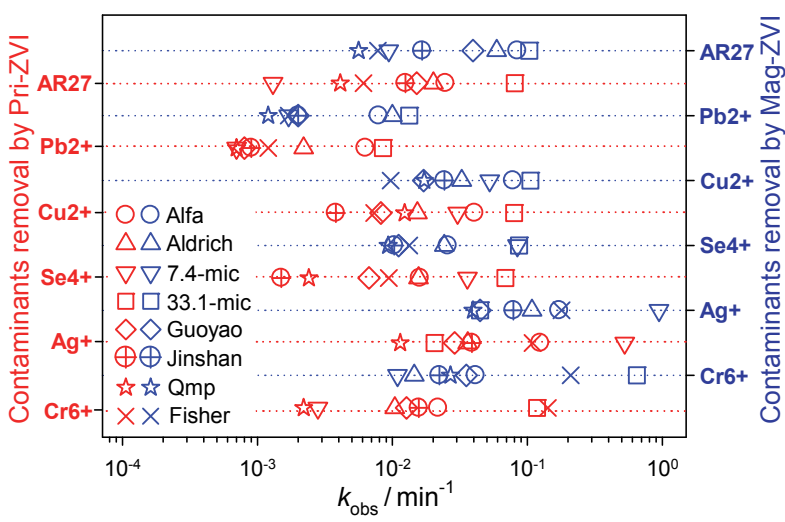

图 8 磁场预磁化对不同来源零价铁去除水中不同污染物表观反应速 率及促进作用概括 ${ }^{[42]}$

Figure 8 Summary of rate constants for $\mathrm{HCrO}_{4}{ }^{-}(\mathrm{Cr} 6+), \mathrm{Ag}^{+}(\mathrm{Ag}+)$, $\mathrm{SeO}_{3}{ }^{2-}(\mathrm{Se} 4+), \mathrm{Cu}^{2+}(\mathrm{Cu} 2+), \mathrm{Pb}^{2+}$ and AR27 sequestration by Pri-ZVI and Mag-ZVI ${ }^{[42]}$

\section{2 磁场预磁化对零价铁除污染的应用可能性的初探}

预磁化能够加速零价铁腐蚀进而促进零价铁对水 中 As 的去除. 因此研究还考察了放置时间对预磁化后 的零价铁除水中 As(III)效果的影响. 研究表明, 相对于 原始零价铁(Pri-ZVI)对水中 As(III)为 $55.0 \%$ 的去除率, Mag-ZVI 在放置时间 $30 \mathrm{~d}$ 后仍能维持一个非常高的活 性，在 $60 \mathrm{~min}$ 内，其对水中 As 去除率只从 $99.5 \%$ 降至 $87.2 \%{ }^{[41]}$. 这一特征对预磁化技术的实际应用有积极作 用. 另外, 为了进一步探究在实际水体中预磁化是否仍 能对零价铁发挥正面效应, 该研究考察了预磁化对零价 铁填充柱去除模拟含砷地下水的影响. 如图 9 所示, 预 磁化能显著延长零价铁填充柱达标处理模拟含 As 地下 水的运行时间，即将零价铁的运行时间从 $22 \mathrm{~h}$ 延长至 $48 \mathrm{~h}$, 预磁化零价铁达标处理原水水样体积是原始零价 铁的 2.3 倍 ${ }^{[41]}$.

基于磁场预磁化强化零价铁对水中污染物的去除 具有操作简单、适用广泛、环境友好、不引起铁颗粒团 聚且维持高活性较长时间等优点, 因而, 零价铁磁场预 磁化是一种应用前景广阔的水污染控制技术.

\section{4 总结与展望}

本系列研究主要围绕探究弱磁场对零价铁去除有 毒污染物及零价铁活化 $\mathrm{H}_{2} \mathrm{O}_{2} / \mathrm{PS}$ 的增效作用及反应机制 展开, 重点考察了弱磁场在提高零价铁反应活性、拓展 零价铁有效 $\mathrm{pH}$ 范围、缓解污染物对零价铁的钝化作用 及恢复已钝化零价铁的反应活性等方面的效能，并探明 了弱磁场在该过程中的作用机制. 为进一步推动弱磁场 强化对零价铁除污染的正面效应, 我们通过预磁化的手 段来改善零价铁的反应活性, 并探究了磁场预磁化对零 价铁除污染的广谱性以及应用的可能性. 


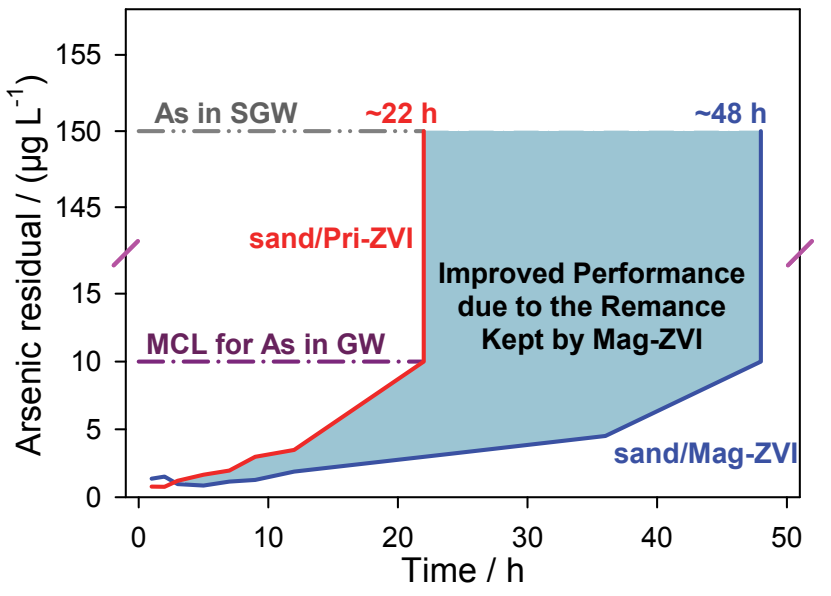

图 9 磁场预磁化对零价铁填充柱除模拟含 As 地下水效果的影响 ${ }^{[41]}$. 图中 MCL 指饮用水卫生标准中 As 的最大限值; SGW 指模拟地下水; GW 指地下水

Figure 9 Arsenic removal from a synthetic groundwater by two column beds packed with sand/Pri-ZVI and sand/Mag-ZVI-500 ${ }^{[41]}$. MCL stands for maximum contaminant level of As; SGW stands for synthetic groundwater; GW stands for groundwater

尽管弱磁场能够提高零价铁的反应活性，但是弱磁 场零价铁体系对有毒金属的电子选择性仍然未知. 考虑 到本研究的主要工作都是在实验室条件下进行, 弱磁场 零价铁水处理技术在实际应用中表现如何尚待进一步 系统地研究. 此外, 虽然预磁化能够明显改善零价铁除 污染的反应活性, 但其对零价铁除污染反应速率的提高 程度有限. 因此, 如何进一步地提升预磁化对零价铁活 性的促进作用有待进一步地探究. 该后续研究的开展可 望进一步推动铁磁水处理技术在有毒金属离子去除方 面的应用, 为水中污染物的高效去除提供新的技术方法 和坚实的理论依据.

\section{作者简介}

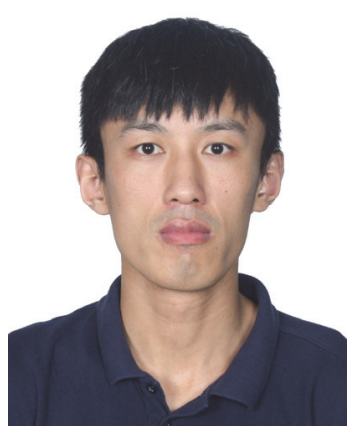

李锦祥, 博士, 导师为关小红教授. 2016 年 12 月毕业于同 济大学市政工程专业, 2017 年 1 月至今在同济大学环境科学与 工程学院从事博士后研究. 主要研究方向为: 弱磁场强化零 价铁对水中污染物的去除效能及其作用机制.

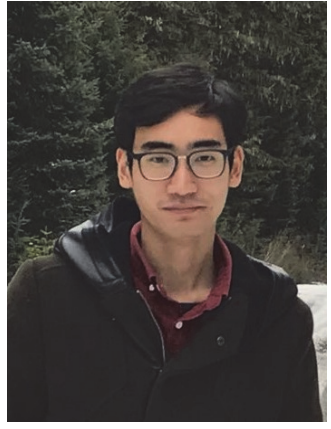

秦荷杰, 博士生, 导师为关小红教授. 2014 年 6 月毕业于 湖南大学给排水科学与工程专业, 2014 年 9 月至今在同济大学 环境科学与工程学院攻读博士研究生. 研究方向为零价铁除 污染过程中的选择性提高方法与机理.

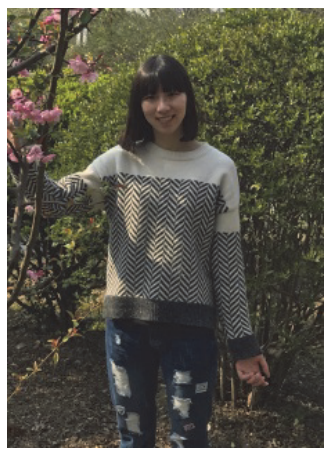

张雪莹, 硕士生, 导师为关小红教授. 2016 年 6 月毕业于 武汉理工大学给水排水工程专业, 2016 年 9 月至今在同济大学 环境科学与工程学院攻读硕士研究生. 主要研究方向为零价 铁对水中污染物的增效新方法及其作用机制.

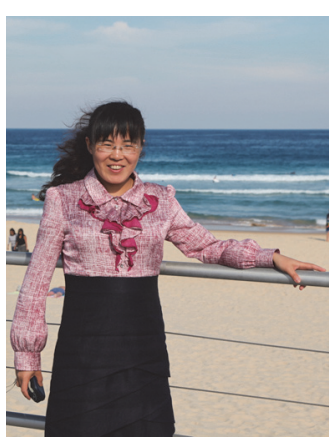

关小红, 同济大学环境科学与工程学院教授、博士生导师, 国家自然科学基金委优秀青年基金获得者，上海市青年科技 启明星计划入选者. 主要从事水污染控制化学方面的研究, 已在 Environ. Sci. Technol.、Water Res.等杂志上发表 SCI 论文 80 余篇, H 因子 25 .

\section{References}

[1] Gould, J. P. Water Res. 1982, 16, 871

[2] Khudenko, B. M. Water Sci. Technol. 1985, 15, 204

[3] Gillham, R. W.; O'Hannesin, S. F. Ground Water 1994, 32, 958.

[4] Matheson, L. J.; Tratnyek, P. G. Environ. Sci. Technol. 1994, 28, 2045.

[5] EPA, USA, Ground Water Remedies Selected at Superfund Sites, 2002.

[6] Guan, X. H.; Sun, Y. K.; Qin, H. J.; Li, J. X.; Lo, I. M.; He, D.; Dong, H. R. Water Res. 2015, 75, 224. 
[7] Wang, C.; Zhang, W. Environ. Sci. Technol. 1997, 94, 9602.

[8] Hung, H. M.; Hoffmann, M. R. Environ. Sci. Technol. 1998, 32, 3011 .

[9] Lien, H. L.; Zhang, W. X. J. Environ. Eng. 1999, 125, 1042.

[10] Harendra, S.; Vipulanandan, C. Colloid Surface A 2008, 322, 6.

[11] Liou, Y. H.; Lo, S. L.; Lin, C. J.; Wen, H. K.; Weng, S. C. J. Hazard. Mater. 2005, 126, 189.

[12] Son, H. S.; Im, J. K.; Zoh, K. D. Water Res. 2009, 43, 1457.

[13] Jou, C. J. G.; Hsieh, S. C.; Lee, C. L.; Lin, C.; Huang, H. W. J. Taiwan Inst. Chem. E 2010, 41, 216.

[14] Xu, J.; Hao, Z.; Xie, C.; Lv, X.; Yang, Y.; Xu, X. Desalination 2012, $284,9$.

[15] Huang, Y. H.; Tang, C.; Zeng, H. Chem. Eng. J. 2012, 200, 257.

[16] Scherer, M. M.; Johnson, K. M.; Westall, J. C.; Tratnyek, P. G. Environ. Sci. Technol. 2001, 35, 2804.

[17] Noubactep, C. Environ. Technol. 2008, 29, 909.

[18] Kim, D. H. J. Hazard. Mater. 2011, 192, 928.

[19] Jiang, J. H.; Li, Y. H.; Cai, W. M. J. Hazard. Mater. 2008, 153, 508.

[20] Ambashta, R. D.; Repo, E.; Sillanpää, M. Ind. Eng. Chem. Res. 2011, 50, 11771 .

[21] Liang, L.; Sun, W.; Guan, X.; Huang, Y.; Choi, W.; Bao, H.; Li, L.; Jiang, Z. Water Res. 2014, 49, 371.

[22] Liang, L.; Guan, X.; Huang, Y.; Ma, J.; Sun, X.; Qiao, J.; Zhou, G. Sep. Purif. Technol. 2015, 156, Part 3, 1064.

[23] Sun, Y. K.; Guan, X. H.; Wang, J. M.; Meng, X. G.; Xu, C. H.; Zhou, G. M. Environ. Sci. Technol. 2014, 48, 6850.

[24] Guan, X.; Jiang, X.; Qiao, J.; Zhou, G. J. Hazard. Mater. 2015, 300, 688.

[25] Jiang, X.; Qiao, J.; Lo, I. M. C.; Wang, L.; Guan, X.; Lu, Z.; Zhou, G.; Xu, C. J. Hazard. Mater. 2015, 283, 880.

[26] Feng, P.; Guan, X. H.; Sun, Y. K.; Choi, W. Y.; Qin, H. J.; Wang, J. M.; Qiao, J. L.; Li, L. N. J. Environ. Sci.-China 2015, 31, 175.
[27] Li, J.; Bao, H.; Xiong, X.; Sun, Y.; Guan, X. Sep. Purif. Technol. 2015, 151, 276

[28] Xu, C.; Zhang, B.; Zhu, L.; Lin, S.; Sun, X.; Jiang, Z.; Tratnyek, P. G. Environ. Sci. Technol. 2016, 50, 1483.

[29] Liang, L. P.; Guan, X. H.; Shi, Z.; Li, J. L.; Wu, Y. N.; Tratnyek, P. G. Environ. Sci. Technol. 2014, 48, 6326.

[30] Xu, H.; Sun, Y.; Li, J.; Li, F.; Guan, X. Environ. Sci. Technol. 2016, 50,8214 .

[31] Xiong, X.; Sun, Y.; Sun, B.; Song, W.; Sun, J.; Gao, N.; Qiao, J.; Guan, X. RSC Adv. 2015, 5, 13357.

[32] Xiang, W.; Zhang, B.; Zhou, T.; Wu, X.; Mao, J. Sci. Rep.-UK 2016, 6.

[33] Xiong, X.; Bo, S.; Jing, Z.; Gao, N.; Shen, J.; Li, J.; Guan, X. Water Res. 2014, 62, 53.

[34] Ragsdale, S. R.; Grant, K. M.; White, H. S. J. Am. Chem. Soc. 1998, 120,13461

[35] Hinds, G.; Coey, J.; Lyons, M. E. G. Electrochem. Commun. 2001, 3, 215.

[36] Lioubashevski, O.; Katz, E.; Willner, I. J. Phy. Chem. B 2004, 108, 5778.

[37] Waskaas, M.; Kharkats, Y. I. J. Electroanal. Chem. 2001, 502, 51.

[38] Li, J.; Qin, H.; Zhang, W.-X.; Shi, Z.; Zhao, D.; Guan, X. Sep. Purif. Technol. 2016, 176, 40 .

[39] Aziz, F.; Pandey, P.; Chandra, M.; Khare, A.; Rana, D. S.; Mavani, K. R. J. Magn. Magn. Mater. 2014, 356, 98.

[40] Ghosh, N.; Mandal, B. K.; Kumar, K. M. J. Magn. Magn. Mater. 2012, 324, 3839.

[41] Li, J. X.; Shi, Z.; Ma, B.; Zhang, P. P.; Jiang, X.; Xiao, Z. J.; Guan, X. H. Environ. Sci. Technol. 2015, 49, 10581.

[42] Li, J.; Qin, H.; Guan, X. Environ. Sci. Technol. 2015, 49, 1440.

[43] Li, X.; Zhou, M.; Pan, Y.; Xu, L. Chem. Eng. J. 2016, 307, 1092.

(Cheng, B.; Fan, Y.) 